\title{
PROBIÓTICO E/OU PREBIÓTICO SOBRE AS CARACTERÍSTICAS DA LEITEGADA E DA MATRIZ LACTANTE
}

\author{
Probiotic and/or prebiotic on litter characteristics and sow breastfeeding \\ Danillo Salgado de Barros ${ }^{1}$, João Garcia Caramori Júnior², Alessandro Luís Fraga, \\ Joadil Gonçalves de Abreu ${ }^{4}$, Valney Souza Correa ${ }^{5}$, Felipe Mainardi ${ }^{5}$
}

\begin{abstract}
RESUMO
Conduziu-se este trabalho, com o objetivo de verificar o efeito do uso de probiótico e/ou prebiótico na alimentação de matrizes suínas. Foi utilizado um delineamento inteiramente casualizado com 4 tratamentos e 6 repetições $(\mathrm{T} 1=$ controle; T2= prebiótico; T3= probiótico; e T4= prebiótico + probiótico), sendo cada fêmea considerada uma parcela. A adição de probiótico e/ou prebiótico diminuiu o número de leitões mumificados. A adição de prebiótico na ração das matrizes adicionado ou não de probiótico, elevou a concentração de proteína no leite no $21^{\circ}$ dia de lactação. No $14^{\circ}$ dia de lactação, as concentrações de Clostridium perfringens foram menores nas fezes de matrizes que se alimentaram de probiótico. No $21^{\circ}$ dia de lactação, as concentrações de Bifidobacterium nas fezes das matrizes foram estatisticamente menores no tratamento com prebiótico (T2). As concentrações de Enterobacteriaceae foram maiores nas fezes de matrizes que receberam a adição de probiótico (T3).
\end{abstract}

Termos para indexação: Fêmea suína, Bacillus subtilis, mananoligossacarideo, intervalo desmame-estro, leite.

\begin{abstract}
The objective of the present study was to in determine the effect of probiotic and/or prebiotic added to feed for sows. An entirely randomized design with 24 sows, 4 treatments and 6 replicates $(\mathrm{T} 1=$ control; $\mathrm{T} 2=$ prebiotic; $\mathrm{T} 3=$ probiotic; and $\mathrm{T} 4=$ prebiotic + probiotic) was used. The addition of probiotic and/or prebiotic decreased the number of mummified piglets. The addition of prebiotic to sow's feed, with or without probiotic, increased the concentration of milk protein on the 21 st day of lactation. On the14th day of lactation, the of Clostridium perfringens was smaller in the faeces of sows fed with probiotic (T3 and T4). On the 21st day of lactation, the concentration of Bifidobacterium in the faeces was statistically lower in sow food with prebiotic (T2). Concentrations of Enterobacteriaceae were higher in the faeces of sows that received probiotic (T3).
\end{abstract}

Index terms: Sow, Bacillus subtilis, mannan oligosaccharides, wean to estrus interval, milk.

(Recebido em 23 de novembro de 2007 e aprovado em 26 de novembro de 2009)

\section{INTRODUÇÃO}

Os antimicrobianos são utilizados como promotores de crescimento a fim de controlar os distúrbios entéricos nas criações intensivas de suínos (FEDALTO et al., 2002). Eles têm melhorado as taxas de crescimento, eficiência alimentar e queda da mortalidade de leitões (ALEXOPOULOS et al., 2004).

Entretanto, esses aditivos vêm sendo alvos de críticas, pois podem promover resistência em bactérias patogênicas ao homem, pela ingestão de carne oriunda de animais que consumiram antibióticos adicionados à dieta (SANCHES et al., 2006). Assim, surge a necessidade de pesquisas em busca de alternativas que substituam os antimicrobianos na promoção do crescimento.
Dentre as alternativas, probióticos e prebióticos têm sido destacados. Os probióticos atuam como auxiliares da recomposição da microbiota intestinal, desfavorecendo a colonização dos microrganismos patogênicos, não deixando resíduos nos produtos de origem animal e não ocasionando resistência às drogas antimicrobianas aos seres humanos, como os antimicrobianos ocasionam (COPPOLA; TURNES, 2004).

Os mecanismos de ação dos probióticos podem ser citados tais como: exclusão competitiva dos sítios adesão, estímulo ao sistema imune (SCHAREK et al., 2005), efeito nutricional, e ainda pela supressão da produção de amônia (SHIM et al., 2005). Diversos trabalhos já foram descritos utilizando os probióticos, sendo a grande maioria realizada em leitões na idade pós-desmame, porém a

\footnotetext{
1Universidade Federal de Mato Grosso/UFMT - Faculdade de Medicina Veterinária/FAMEV - Av. Fernando Corrêa da Costa, s/n. - Bairro Coxipó - 78060-900 Cuiabá, MT - danillovet@hotmail.com

2Universidade Federal de Mato Grosso/UFMT - Faculdade de Medicina Veterinária/FAMEV - Departamento de Produção Animal - Cuiabá, MT ${ }^{3}$ Universidade Federal de Mato Grosso/UFMT - Faculdade de Medicina Veterinária/FAMEV - Departamento de Ciências Biológicas/ Zootecnia - Cuiabá, MT ${ }^{4}$ Universidade Federal de Mato Grosso/UFMT - Faculdade de Medicina Veterinária/FAMEV - Departamento de Zootecnia e Extensão Rural - Cuiabá, MT ${ }^{5}$ Universidade Federal de Mato Grosso/UFMT - Faculdade de Medicina Veterinária/FAMEV - Cuiabá, MT
} 
utilização dessas cepas probióticas, em rações de matrizes em fase de gestação e lactação, ainda é pouco estudada.

Zani et al. (1998) compararam a utilização do probiótico Bacillus cereus ao antibiótico furalozidone adicionados à dieta de matrizes nos cinco últimos dias de gestação ao $21^{\circ}$ dia de lactação. Esses pesquisadores verificaram que não houve diferença na contagem de Escherichia coli no intestino de matrizes suínas. Lázaro et al. (2005) utilizaram como probiótico as cepas Saccharomyces cerevisiae, Bacillus subtilis e Bacillus coagulans, em matrizes no último terço da gestação e durante a lactação, porém não observaram diferença quanto ao consumo de ração em relação ao grupo controle.

Prebióticos, por sua vez, são definidos como carboidratos não digeríveis por enzimas (SAAD, 2006), sais ou ácidos produzidos pelo organismo animal (AVCARE, 2003). O mananoligossacarídeo (MOS) é um exemplo de prebiótico. Este possui característica adsortiva ao atuar nos sítios de aderência D-manose dos patógenos gram-negativos, eliminando-os nas fezes, evitando assim a colonização da microbiota patógena no epitélio intestinal (KOGAN; KOCHER, 2007).

Pesquisa de Sanches et al. (2006) mostrou que o MOS, quando utilizado em leitões pós-desmamados, promoveu mesmo desempenho quando comparados com o uso de probiótico (Bacillus subtilis), antibiótico (olanquidox) e em associação ao probiótico.

O desenvolvimento de uma microbiota favorável depende da presença de nutrientes e condições ambientais adequadas, assim ao se fornecer prebióticos, esse irá potencializar o efeito do probiótico no organismo do hospedeiro (COPPOLA; TURNES, 2004). Tal afirmação foi observada por Shim et al. (2005) que relatou o melhor desempenho de leitões desmamados com fornecimento dos probióticos Bifidobacterium, Lactobacillus, Bacillus e Aspergillus associado ao prebiótico frutoligossacarídeo, além da concentração de Lactobacillus no intestino delgado ter sido maior que a observada nos animais que receberam os aditivos isolados.

Nesse contexto, conduziu-se este estudo, com o objetivo de verificar o efeito da utilização de probiótico e/ ou prebiótico, na alimentação de matrizes entre a fase final de gestação e a fase de lactação, sobre o desempenho reprodutivo, zootécnico, características físico-químicas do leite e microbiologia das fezes das matrizes.

\section{MATERIAL E MÉTODOS}

\section{Local do Experimento, Instalações e Animais}

$\mathrm{O}$ experimento foi conduzido em um sistema de produção de suínos situado no Médio Norte do Estado de
Mato Grosso, no período de março a junho de 2007. Foram utilizadas 24 matrizes de mesma linhagem, com três partos, transferidas para a maternidade seis dias antes do parto. As duas salas utilizadas possuíam 18 celas de parição suspensas $(2,00 \mathrm{~m} \times 1,50 \mathrm{~m})$ cada, dotadas de comedouros de concreto para a matriz, comedouro para os leitões, bebedouros tipo chupeta específicas para matriz e leitões, área de escape para os leitões, escamoteador com lâmpada incandescente, ambiente controlado por meio de cortinas. As salas foram limpas previamente com jato de água e desinfetadas com amônia quaternária. Em seguida, foi passada lança chamas e feita a fumigação com formol. Realizou-se vazio sanitário de dois dias. No ato da transferência, as matrizes foram higienizadas com amônia quaternária. Durante o experimento, os animais receberam iguais condições de manejo. No $14^{\circ}$ dia de lactação, as fêmeas receberam a vacina tríplice (leptospirose, parvovirose e erisipela). As temperaturas mínimas e máximas foram $24,53 \pm 0,64^{\circ} \mathrm{C}$ e $32,52 \pm 1,57^{\circ} \mathrm{C}$, respectivamente.

Utilizou-se delineamento inteiramente casualizado com quatro tratamentos e seis repetições. Os tratamentos experimentais foram:

- $\mathrm{T} 1=$ Controle (Dieta basal= milho, farelo de soja, fosfato bicálcico, calcário, óleo vegetal, sal, antioxidante, mistura mineral e vitamínica; fornecida rotineiramente na granja, isenta de aditivos antimicrobianos e elaborada por assistência técnica com níveis de 17,2\% de PB e $3.400 \mathrm{kcal} /$ $\mathrm{kg}$ de $\mathrm{ED})$;

- T2= Dieta basal + prebiótico;

- T3 = Dieta basal + probiótico; e

- T4= Dieta basal + prebiótico e probiótico.

Os tratamentos corresponderam a dietas fornecidas às matrizes seis dias antes do parto até o $21^{\circ}$ dia de lactação. O prebiótico utilizado foi o mananoligossacarídeo (MOS), extraído da parede celular externa da levedura Saccharomyces cerevisiae na dosagem de $1,5 \mathrm{~kg} / \mathrm{t}$ de ração. O probiótico utilizado foi o Bacillus subtilis $\left(1 \times 10^{10} \mathrm{UFC} /\right.$ grama de produto), na dosagem de $1,0 \mathrm{~kg} / \mathrm{t}$ durante os seis últimos dias de gestação; $0,5 \mathrm{~kg} / \mathrm{t}$ do dia do parto até o $10^{\circ}$ dia de lactação; e $0,1 \mathrm{~kg} / \mathrm{t}$ do $11^{\circ}$ ao $21^{\circ}$ dia de lactação, conforme recomendação do fabricante. $\mathrm{Na}$ associação prebiótico/ probiótico, as mesmas concentrações foram mantidas. Cada unidade experimental foi composta por uma matriz.

Na sala 1, foram alojadas as matrizes que não receberam probiótico na dieta (T1 e $\mathrm{T} 2$ ), pois estas foram alojadas na sala 2 (T3 e T4), para evitar a contaminação cruzada com os esporos do Bacillus subtilis. Foram utilizados baldes para arraçoamento, vassouras, rodos e 
pás de limpar fezes para cada tratamento, cal virgem e desinfetante à base de cresol, na porta de cada sala de maternidade. As matrizes foram arraçoadas com ração lactação, desde seis dias antes do parto até o final da fase de lactação (21 dias), duas vezes ao dia, sendo fornecido $3,0 \mathrm{~kg}$ às $6: 00 \mathrm{~h}$ e $3,0 \mathrm{~kg}$ às 18:00 h. Antes do trato, adicionava-se 3,0 L de água (1:1).

Ao parto foram registrados o número de leitões nascidos vivos, natimortos e mumificados. Durante a fase de aleitamento, foram registrados o número de leitões mortos para posterior determinação da taxa da mortalidade no pré-desmame e número de leitões desmamados.

Após o parto, até o $21^{\circ}$ dia de lactação, foi realizada a pesagem da sobra da ração após cada arraçoamento (manhã e tarde), para se determinar o consumo de ração de cada matriz.

Realizou-se a avaliação microbiológica das fezes de cada matriz colhida em três momentos: dia 0 (zero) (antes do período de fornecimento dos aditivos), no $14^{\circ}$ e $21^{\circ}$ dia de lactação. A coleta foi realizada através da via transretal com auxílio de luva de procedimento individual para cada matriz. As fezes foram acondicionadas em sacolas plásticas, previamente identificadas, acondicionadas em isopor com gelo reciclável e enviadas imediatamente ao laboratório da empresa Uniquímica, localizada em São Paulo-SP, onde foram analisadas, de acordo com Tomotari et al. (1976), em menos de 24 horas. As bactérias isoladas foram Lactobacillus, Bifidobacterium, Bacillus subtilis, Enterobacteriaceae, Bactérias anaeróbias totais e Clostridium perfringens.

Amostras de leite das matrizes foram coletadas no $14^{\circ}$ e $21^{\circ}$ dia de lactação para proceder análises físicoquímicas. Para esse procedimento, os leitões foram retirados da matriz pelo período de quatro horas e os tetos foram limpos com papel toalha. Foram administradas na matriz, $2 \mathrm{~mL}$ de ocitocina (10 UI/mL) por via endovenosa. Em seguida, foi feita ordenha individual e o leite recolhido em frasco contendo uma pastilha de Bronopol (conservante), foi acondicionado em isopor com gelo reciclável e enviado ao Laboratório Estadual da Qualidade do Leite, situado em Concórdia SC, chegando com temperatura em torno de $7^{\circ} \mathrm{C}$. Cada amostra continha aproximadamente $50 \mathrm{~mL}$. Foram realizadas análises de gordura, proteína, lactose, sólidos totais e contagem de células somáticas por infravermelho, utilizando os equipamentos Bentley e Somacount ${ }^{\circledR}$.

Os leitões foram desmamados no $21^{\circ}$ dia de lactação e as matrizes foram transferidas para o galpão de gestação. Neste local, elas foram verificadas diariamente, com auxílio de um cachaço, para identificação do estro e determinado o intervalo desmame-estro. Quando o estro foi diagnosticado, as matrizes foram inseminadas 12,24 e 36 horas após a detecção. Do $18^{\circ}$ ao $21^{\circ}$, após a inseminação, foram novamente testadas para registrar eventual retorno ao estro.

Os dados de número de leitões nascidos vivos, natimortos, mumificados, desmamados, mortalidade prédesmame e contagem de células somáticas do leite foram transformados em $\mathrm{y}=\sqrt{x+5}$ e os dados da análise microbiológica das fezes foram transformados em logarítimo de 10, conforme Barbin (2003). Os dados foram submetidos à análise de variância e as médias comparadas pelo teste Scott-Knott a 5\% de probabilidade, utilizando o Software SAEG (1993).

\section{RESULTADOS E DISCUSSÃO}

Os resultados obtidos para o número de leitões nascidos vivos, natimortos, desmamados e a taxa de mortalidade pré-desmame não diferiram estatisticamente ( $\mathrm{P}>0,05)$ entre si (Tabela 1). Resultados semelhantes também foram observados por Alexopoulos et al. (2004), com cepas probióticas de Bacillus subtilis e B. licheniformis, exceto para número de leitões desmamados e taxa de mortalidade que foram, respectivamente, maior e menor no grupo tratado com probiótico em relação ao grupo controle. Essa diferença pode ser explicada em função das cepas utilizadas, ao contrário da presente pesquisa, que utilizou somente o Bacillus subtilis. Outro fato a ser considerado foi o fornecimento do probiótico 14 dias antes do parto, enquanto no presente trabalho ocorreu a partir de seis dias.

O número de leitões mumificados foi significativamente maior $(\mathrm{P}<0,05)$ nas matrizes que receberam o tratamento controle (Tabela 1), resultados contraditórios a Alexopoulos et al. (2006) quando utilizou Bacillus toyoi.

Os resultados obtidos para o consumo médio de ração durante o experimento estão descritos na Tabela 2. Não houve diferença significativa $(P>0,05)$ entre os tratamentos utilizados. Lázaro et al. (2005), ao utilizarem as cepas de Saccharomyces cerevisiae, Bacillus subtilis $e$ Bacillus coagulans, também não verificaram diferença. No entanto, Alexopoulos et al. (2004) observaram nos primeiros 14 dias de lactação, maior consumo de ração das matrizes que receberam probióticos na ração. $\mathrm{O}$ consumo das matrizes não foi diferente entre os tratamentos, pois o fornecimento das dietas 6 dias antes do parto não é capaz de influenciar totalmente a microbiota intestinal e causar seus efeitos. 
Tabela 1 - Características de leitões provenientes de matrizes dentre os diferentes tratamentos.

\begin{tabular}{lccccc}
\hline \multicolumn{2}{c}{ Ração esperimental } & & & \\
Características & Controle & MOS & Bacillus subtilis & $\begin{array}{c}\text { MOS+ Bacillus } \\
\text { subtilis }\end{array}$ & CV (\%) \\
\hline Nascidos vivos & 11,33 & 12,53 & 12,10 & 11,26 & 11,1 \\
Natimortos & 1,16 & 0,54 & 0,80 & 0,84 & 37,8 \\
Mumificados & $0,99 \mathrm{~b}$ & $0,12 \mathrm{a}$ & $0,12 \mathrm{a}$ & $0,0 \mathrm{a}$ & 32,0 \\
Desmamados & 9,55 & 10,72 & 10,32 & 10,32 & 8,2 \\
Mortalidade pré & 1,57 & 1,44 & 1,47 & 0,83 & 38,6 \\
desmame $(\%)$ & & & & \\
\hline
\end{tabular}

${ }^{1}$ Médias seguidas de letras diferentes, na mesma linha, diferem pelo teste Scott-Knott $(\mathrm{P}<0,05)$.

Tabela 2 - Consumo médio de ração $(\mathrm{kg})$ das matrizes na fase de lactação submetidas aos diferentes tratamentos.

\begin{tabular}{lccccc}
\hline \multicolumn{2}{c}{ Ração experimental } & & & \\
Período & Controle & MOS & Bacillus subtilis & $\begin{array}{c}\text { MOS+ Bacillus } \\
\text { subtilis }\end{array}$ & CV (\%) \\
\hline $0-7$ dias & 54,76 & 55,10 & 59,71 & 57,93 & 21,22 \\
$8-14$ dias & 73,17 & 71,00 & 72,90 & 76,40 & 12,96 \\
$15-21$ dias & 69,18 & 64,35 & 68,86 & 74,42 & 10,48 \\
Total & 192,38 & 212,72 & 220,16 & 230,29 & 13,30 \\
\hline
\end{tabular}

${ }^{1}$ As médias não diferiram pelo teste Scott-knott $(\mathrm{P}>0,05)$.

Os resultados da análise do leite das matrizes encontram-se na Tabela 3. Aos 14 dias de lactação não foram encontradas diferenças $(\mathrm{P}>0,05)$ com relação à gordura, proteína, lactose, sólidos totais e contagem de células somáticas. Entretanto, nos ensaios de Alexopoulos et al. (2004), houve maior concentração de proteína e gordura no leite das matrizes no $14^{\circ}$ dia de lactação quando tratadas com probióticos a base de Bacillus subtilis $e$ Bacillus toyoi. Esses autores concluíram que o maior consumo de ração das matrizes tratadas com probióticos em relação ao grupo controle, promoveu maior aporte de nutrientes no período de lactação.

No $21^{\circ}$ dia de lactação, foi observada maior percentagem $(\mathrm{P}<0,05)$ de proteína no leite de matrizes que consumiram MOS e MOS + Bacillus subtilis como aditivo de ração (Tabela 3). A adição de MOS, com ou sem Bacillus subtilis na ração das matrizes, promoveu a secreção de leite mais protéico ao final da fase de lactação, o que pode proporcionar maior desempenho dos animais lactentes. Jurgens et al. (1997) observaram diferentes resultados ao utilizar a levedura Saccharomyces cerevisiae como probiótico em matrizes no final da gestação, até o $21^{\circ}$ dia de lactação, pois verificaram maiores concentrações de gordura e sólidos totais no leite aos 21 dias de lactação.
Além disso, obtiveram maiores concentrações de proteína e imunoglobulina (Ig) no leite neste momento. Dessa forma, torna-se importante a utilização de MOS na dieta de matrizes para aumentar proteína do leite, principalmente em sistemas com desmame mais tardios, como a União Européia preconiza para proporcionar o bem-estar animal.

Kamimura et al. (2006) ao tratarem leitões do desmame aos 63 dias de idade com MOS associado ao promotor colistina obtiveram maiores concentrações de Ig tipo A no soro dos leitões, à descreche.

Os resultados das análises microbiológicas das fezes das matrizes encontram-se na Tabela 4. No dia zero, as concentrações de Bacillus subtilis foram iguais a zero para todos os tratamentos e estas não foram levadas em consideração nas análises estatísticas. Seus valores encontraram-se abaixo de 2,3 (escala log). Ainda no dia zero, as concentrações de Lactobacillus, Bifidobacterium, Enterobacteriaceae e Bactérias Anaeróbias Totais não diferiram entre si $(\mathrm{P}>0,05)$, como era esperado, uma vez que, não havia efeito dos tratamentos.

Nas análises de fezes das matrizes suínas, realizadas no $14^{\circ}$ e $21^{\circ}$ dia de lactação, as concentrações de Bacillus subtilis foram maiores $(\mathrm{P}<0,05)$ nas amostras de fezes das fêmeas que receberam Bacillus subtilis, associado ou não 
Tabela 3 - Composição físico-química do leite das matrizes suínas no $14^{\circ}$ e $21^{\circ}$ dia de lactação, submetidas aos diferentes tratamentos.

\begin{tabular}{|c|c|c|c|c|}
\hline \multirow[b]{2}{*}{ Parâmetros } & \multicolumn{4}{|c|}{ Tratamentos $^{1}$} \\
\hline & Controle & MOS & Bacillus subtilis & MOS+ Bacillus subtilis \\
\hline \multicolumn{5}{|c|}{ Aos 14 dias } \\
\hline Gordura (\%) & 4,78 & 5,23 & 3,96 & 5,55 \\
\hline Proteína (\%) & 5,17 & 5,10 & 4,79 & 4,76 \\
\hline Lactose $(\%)$ & 5,20 & 5,00 & 5,48 & 5,35 \\
\hline Sólidos totais (\%) & 16,08 & 16,22 & 15,24 & 16,61 \\
\hline \multicolumn{5}{|l|}{ Contagem de Células } \\
\hline $\begin{array}{c}\text { Somáticas } \\
\left(\times 10^{3}\right)\end{array}$ & 5,80 & 6,33 & 5,61 & 6,03 \\
\hline \multicolumn{5}{|c|}{ Aos 21 dias } \\
\hline Gordura (\%) & 5,60 & 5,12 & 4,61 & 5,06 \\
\hline Proteína (\%) & $5,03 \mathrm{~b}$ & $5,67 \mathrm{a}$ & $4,84 \mathrm{~b}$ & $5,37 \mathrm{a}$ \\
\hline Lactose $(\%)$ & 5,44 & 5,30 & 5,64 & 5,42 \\
\hline Sólidos totais (\%) & 17,22 & 17,11 & 16,16 & 16,90 \\
\hline Contagem de Células & & & & \\
\hline $\begin{array}{c}\text { Somáticas } \\
\left(\times 10^{3}\right)\end{array}$ & 5,54 & 5,89 & 5,40 & 5,68 \\
\hline
\end{tabular}

${ }^{1}$ Médias seguidas de letras diferentes, na mesma linha, diferem pelo teste Scott-Knott $(\mathrm{P}<0,05)$.

ao MOS, quando comparadas com as amostras do grupo controle e daquelas que receberam apenas MOS (Tabela 4). Esses resultados reforçam as idéias de Coppola e Turnes (2004) os quais citam que, embora o Bacillus subtilis não colonize o TGI, por não serem habitantes normais da microbiota dos suínos, esse atinge a luz intestinal com um maior número de microrganismos viáveis, quando comparado aos probióticos de bactérias ácido-láticas como Lactobacillus acidofilus e Pediococcus acidilactici. Também é importante salientar que as amostras que não receberam Bacillus subtilis (T1 e T2) via dieta apresentaram pouca concentração de Bacillus subtilis nas fezes, demonstrando, com isso, que o controle durante o experimento foi efetivo entre os tratamentos, no intuito de impedir a contaminação cruzada entre estes.

Os resultados da análise de fezes no $14^{\circ}$ dia de lactação demonstraram que não houve diferença $(\mathrm{P}>0,05)$ com relação às concentrações de Lactobacillus, Bifidobacterium, Enterobacteriaceae e Bactérias Anaeróbias Totais (Tabela 4). Entretanto, encontrou-se efeito benéfico $(\mathrm{P}<0,05)$ nas concentrações de Clostridium perfringens, nos quais as fêmeas suplementadas com Bacillus subtilis e aquelas com MOS + Bacillus subtilis apresentaram menores concentrações desse agente patogênico em relação ao tratamento controle e ao MOS. A concentração de Clostridium perfringens foi menor $(\mathrm{P}<0,05)$ no grupo tratado com MOS com relação ao grupo controle, conforme demonstra a Tabela 4. Os resultados da presente pesquisa (in vivo) estão de acordo com Yeow e Hai (2005), aos quais demonstraram que o Bacillus subtilis "in vitro", apresentou atividade inibitória ao Clostridium perfringens.

Com relação às fezes das matrizes analisadas no $21^{\circ}$ dia de lactação, não houve diferença significativa $(\mathrm{P}>0,05)$ entre os diferentes tratamentos nas concentrações de Lactobacillus, Bactérias anaeróbias totais e Clostridium perfringens.

As concentrações de Bifidobacterium foram menores $(\mathrm{P}<0,05)$ nas fezes de matrizes que receberam a adição de MOS em relação aos demais tratamentos experimentais ao $21^{\circ}$ dia de lactação (Tabela 4). Diferentes resultados foram encontrados por Moura et al. (2007) que ao utilizar o prebiótico xilo-oligossacarídeo (XOS) associado ao probiótico Saccharomyces cerevisiae, em leitões desmamados, não verificaram mudanças nas concentrações intestinais de Lactobacillus e Bifidobacterium, quando comparado ao tratamento somente com prebiótico que promoveu melhorias nas concentrações de Lactobacillus. No entanto, esses autores utilizaram esses aditivos apenas em leitões desmamados e não matrizes. 
As concentrações de Enterobacteriaceae nas fezes das matrizes que receberam MOS + Bacillus subtilis foram maiores $(\mathrm{P}<0,05)$ em relação aos demais tratamentos. Wang et al. (2006), em estudo com ratos, compararam o prebiótico alginate ao frutoligossacarídeo (FOS), concluindo que o alginate aumentou a concentração de Bifidobacterium e promoveu maior inibição às concentrações de Enterobacteriaceae, quando comparado ao FOS, demonstrando variações entre os oligossacarídeos.
Com relação ao intervalo desmame-estro, não houve efeito das dietas experimentais $(\mathrm{P}>0,05)$ (Tabela 5). Resultados semelhantes foram encontrados por Alexopoulos et al. (2004), com a utilização de Bacillus subtilis e Bacillus licheniformis; Alexopoulos et al. (2006), com Bacillus toyoi; Jurgens et al. (1997), utilizando a adição de Saccharomyces cerevisiae. Esses autores trabalharam com esses aditivos em final de gestação e durante a fase de lactação de matrizes suínas.

Tabela 4 - Resultados da análise microbiológica das fezes (log) das matrizes antes do início dos tratamentos, 14 e 21 dias de lactação, submetidas aos diferentes tratamentos de aditivos de ração.

\begin{tabular}{|c|c|c|c|c|c|}
\hline & \multicolumn{4}{|c|}{ Tratamentos $^{1}$} & \multirow[b]{2}{*}{$\mathrm{CV}(\%)$} \\
\hline & Controle & MOS & Bacillus subtilis & $\begin{array}{c}\text { MOS }+ \text { Bacillus } \\
\text { subtilis }\end{array}$ & \\
\hline & \multicolumn{5}{|c|}{ Dia zero } \\
\hline Lactobacillus & 8,42 & 8,18 & 7,82 & 8,36 & 7,81 \\
\hline Bifidobacterium & 7,51 & 7,73 & 7,74 & 7,30 & 6,21 \\
\hline Bacillus subtilis $^{2}$ & n.i. & n.i. & n.i. & n.i. & - \\
\hline Enterobacteriaceae & 7,82 & 8,05 & 7,90 & 7,77 & 4,24 \\
\hline Clostridium perfringens $^{2}$ & n.i. & n.i. & n.i. & n.i. & - \\
\hline \multirow[t]{2}{*}{ Bactérias anaeróbias totais } & 9,38 & 9,49 & 9,26 & 9,13 & 3,26 \\
\hline & \multicolumn{5}{|c|}{ Aos 14 dias } \\
\hline Lactobacillus & 8,54 & 8,43 & 8,11 & 8,40 & 8,21 \\
\hline Bifidobacterium & 6,78 & 7,16 & 7,10 & 6,74 & 12,27 \\
\hline Bacillus subtilis & $2,76 \mathrm{~b}$ & $3,32 \mathrm{~b}$ & $6,52 \mathrm{a}$ & $6,51 \mathrm{a}$ & 12,70 \\
\hline Enterobacteriaceae & 7,21 & 7,36 & 7,44 & 7,60 & 4,07 \\
\hline Clostridium perfringens & 9,09 a & $7,36 \mathrm{~b}$ & $5,89 \mathrm{c}$ & $5,94 \mathrm{c}$ & 14,66 \\
\hline \multirow[t]{2}{*}{ Bactérias anaeróbias totais } & 8,84 & 9,11 & 8,79 & 9,10 & 3,70 \\
\hline & \multicolumn{5}{|c|}{ Aos 21 dias } \\
\hline Lactobacillus & 8,33 & 7,90 & 7,83 & 7,85 & 8,03 \\
\hline Bifidobacterium & $8,53 \mathrm{a}$ & $7,55 \mathrm{~b}$ & $8,51 \mathrm{a}$ & $8,24 \mathrm{a}$ & 6,92 \\
\hline Bacillus subtilis & $2,30 \mathrm{~b}$ & $2,30 \mathrm{~b}$ & $6,23 \mathrm{a}$ & $6,45 \mathrm{a}$ & 4,74 \\
\hline Enterobacteriaceae & $7,07 \mathrm{~b}$ & $7,13 \mathrm{~b}$ & $7,30 \mathrm{~b}$ & $7,94 \mathrm{a}$ & 4,70 \\
\hline Clostridium perfringens & 5,92 & 5,57 & 5,57 & 6,73 & 31,67 \\
\hline Bactérias anaeróbias totais & 9,50 & 9,17 & 9,27 & 8,99 & 5,22 \\
\hline
\end{tabular}

${ }^{1}$ Médias deguidas de letras diferentes, num mesmo período e na mesma linha, diferem entre si pelo teste de Scott - Knott $(\mathrm{P}<0,05)$. ${ }^{2}$ n.i. $=$ não isolado.

Tabela 5 - Intervalo desmame-estro (IDE) das matrizes suínas submetidas aos diferentes tratamentos de aditivos de ração.

\begin{tabular}{cccccc}
\hline & \multicolumn{5}{c}{ Tratamentos } \\
\cline { 2 - 5 } & Controle & MOS & Bacillus subtilis & $\begin{array}{c}\text { MOS + } \\
\text { Bacillus subtilis }\end{array}$ & CV (\%) \\
\hline IDE (dias) & 2,08 & 2,40 & 2,11 & 1,96 & 18,46 \\
\hline
\end{tabular}


No presente estudo, das 24 matrizes pesquisadas, nenhuma retornou ao estro pós-inseminação artificial. Por outro lado, Alexopoulos et al. (2004), ao utilizarem Bacillus subtilis e Bacillus licheniformis em matrizes suínas antes do parto, ao final da lactação, identificaram maior taxa de retorno ao estro no tratamento controle que não foi utilizado nenhum aditivo.

\section{CONCLUSÕES}

Houve diminuição no número de leitões mumificados quando as matrizes foram tratadas com Bacillus subtilis e/ou MOS na dieta.

Matrizes tratadas com MOS tiveram leite com maior teor de proteína no $21^{\circ}$ dia de lactação.

A adição de probiótico aumentou as concentrações de Bacillus subtilis durante a lactação e diminuiu Clostridium perfringens, no $14^{\circ}$ dia de lactação, nas fezes das matrizes.

As concentrações de Bifidobacterium foram menores nas fezes de matrizes ao $21^{\circ}$ dia de lactação quando são tratadas somente com MOS na ração.

A adição de MOS + Bacillus subtilis na ração de fêmeas suínas aumentaram as concentrações de Enterobacteriaceae nas fezes, aos $21^{\circ}$ dia de lactação.

\section{REFERÊNCIAS BIBLIOGRÁFICAS}

ALEXOPOULOS, C. etal. Field evaluation of the efficacy of a probiotic containing Bacillus licheniformis and Bacillus subtilis spores, on the health status and performance of sows and their litters. Journal of Animal Physiology and Animal Nutrition, Athens, v.88, p.381-392, 2004.

ALEXOPOULOS, C. et al. Probiosis in sows by administration of Bacillus toyoi spores during late pregnancy and lactation: effect on their health status/ performance and on litter characteristics. International Journal of Probiotics and Prebiotics, Athens, v.1, n.1, p.33-40, 2006.

AVCARE. The role of enteric antibiotics in livestock production. Canberra: Advanced Veterinary Therapeutics, 2003.

BARBIN, D. Planejamento e análise estatística de experimentos agropecuários. Arapongas: Midas, 2003. $194 \mathrm{p}$.

COPPOLA, M.M.; TURNES, C.G. Probióticos e resposta imune. Ciência Rural, Santa Maria, v.34, n.4, p.12971303, jul./ago. 2004.
FEDALTO, L.M.; TKACZ, M.; ADER, L.P. Probióticos na alimentação de leitões do desmame aos 63 dias de idade. Archives of Veterinary Science, v.7, n.1, p.83-88, 2002.

JURGENS, M.H.; RIKABI, R.A.; ZIMMERMAN, D.R. The effect of dietary active dry yeast supplement on performance of sows during gestation-lactation and their pigs. Journal of Animal Science, Baltimore, v.75, p.593597, 1997.

KAMIMURA, R. et al. Efeitos de mananoligossacarídeo e colistina sobre a histomorfometria intestinal e níveis de IgA e IgG séricas em leitões. Veterinária Notícias, v.12, n.2, p.153-160, dez. 2006.

KOGAN, G.; KOCHER, A. Role of yeast cell wall polysaccharides in pig nutrition and health protection. Livestock Science, v.109, p.161-165, 2007.

LÁZARO, C.D. et al. Efecto de probióticos en el alimento de marranas sobre los parámetros productivos de lechones. Revista de Investigaciones Veterinarias del Perú, Lima, v.16, n.2, p.97-102, 2005.

MOURA, P. et al. Effect of xylo-oligosaccharides from corn cobs autohydrolysis on the intestinal microbiota of piglets after weaning. Livestock Science, Amsterdam, n.108, p.244-248, 2007.

SAAD, S.M.I. Probióticos e prebióticos: o estado da arte. Brazilian Journal of Pharmaceutical Sciences, São Paulo, v.42, n.1, p.1-16, jan./mar. 2006.

SANCHES, A.L. et al. Utilização de probioticos, prebióticos e simbioticos em rações de leitões ao desmame. Ciência e Agrotecnologia, Lavras, v.30, n.4, p.774-777, jul./ago. 2006.

SHIM S.B. et al. Effects of feeding antibiotic-free creep feed supplemented with oligofructose, probiotics or synbiotics to suckling piglets increases the preweaning weight gain and composition of intestinal microbiota. Archives of Animal Nutrition, The Netherlands, v.59, p.419-427, 2005.

SCHAREK L. et al. Influence of a probiotic Enterococcus faecium strain on development of the immune system of sows and piglets. Veterinary Immunology and Immunopathology, Berlin, v.105, p.151161, 2005. 
TOMOTARI, M. et al. Zbl. Bakt. Hyg., v.A234, p.219233, 1976.

UNIVERSIDADE FEDERAL DE VIÇOSA. Sistema de Análises Estatísticas e Genéticas. Versão 5.0. Viçosa, MG, 1993.

WANG, Y. et al. In vivo prebiotic properties of alginate oligosaccharides prepared through enzymatic hydrolysis of alginate. Nutrition Research, v.26, p.597-603, 2006.
YEOW, L.T.A.; HAI, M.T. Inhibition of Clostridium perfringens by a novel strain of Bacillus subtilis isolated from the gastrointestinal tracts of healthy chickens. Applied and Environmental Microbiology, v.71, n.8, p.4185-4190, 2005.

ZANI, J.L. et al. Effects of probiotic CenBiot on the controlo $f$ diarrhoea and feed efficiency in pigs.

Journal of Applied Microbiology, Pelotas, v.84, p.68-71, 1998. 